\title{
Excesso de peso e deslocamento para a escola em adolescentes de Uruguaiana/RS
}

\section{Overweight and commuting to school in adolescents from the city of Uruguaiana/RS, Brazil}

\author{
Anne Ribeiro Streb', Susane Graup', Mauren Lúcia de Araújo Bergmann', Gabriel Gustavo Bergmann
}

\section{Resumo}

O objetivo deste estudo foi identificar as associações entre a forma de deslocamento até a escola e frequência de excesso de peso em adolescentes. Este estudo descritivo foi realizado com 1.455 escolares (50,9\% do sexo feminino) de 10 a 17 anos selecionados aleatoriamente em múltiplas etapas. Para caracterizar o deslocamento à escola utilizou-se um questionário em que os alunos indicaram a forma de transporte até a mesma (ativo ou passivo). O excesso de peso foi considerado pela categorização do índice de massa corporal por proposta internacional. Os dados foram descritos frequências absolutas e relativas e o intervalo de confiança de $95 \%$. As análises de associação bruta foram realizadas pelo teste Qui-Quadrado. As análises ajustadas foram realizadas pela regressão logística binária e a magnitude das associações foi medida pela Odds Ratio (OR). O nível de significância adotado foi de 5\%. Do total da amostra, 75,7\% (IC95\%: 73,5-77,9) apresentou deslocamento ativo até a escola e 27,8\% (IC95\%: 25,4-30,2) foi classificado com excesso de peso. A análise de regressão, com ajuste para sexo, idade, nível socioeconômico e zona de moradia, indicou que adolescentes que realizam o deslocamento para a escola de forma passiva tem mais chance (OR:1,50; IC95\%:1,12-2,01) de apresentarem excesso de peso. Quando a análise é estratificada por sexo, a associação permanece significativa apenas entre as meninas $(\mathrm{p}<0,05)$. Os resultados obtidos sugerem que realizar deslocamento passivo até a escola está associado ao excesso de peso em adolescentes.

\section{Palavras-chave}

Índice de massa corporal; Sedentarismo; Jovens.

\begin{abstract}
The aim of this study was to identify the association between commuting to school and overweight and the frequency of obesity and in adolescents. This descriptive study was conducted with 1,455 adolescents (50.9\% female) aged 10 to 17 years randomly selected. Commuting to school was assessed using adolescents' self -report about the way they usually go to school (active or passive). Overweight and obesity were considered by classification of the body mass index using an international cut offs. Absolute and relative frequency and the confidence interval of 95\% (CI95\%) were used to describe the sample. Crude associations were tested by Chi -square test. Adjusted analyses were conducted using binary logistic regression and the magnitude of associations was measured by Odds Ratio (OR). Adopted significance level was 5\%. In the entire sample, 75.7\% (IC95\%: 73.5-77.9) showed active commuting to school and 27.8\% (IC95\%: 25.4-30.2) were overweight/obese. Regression analyze adjusted by gender, age, socioeconomic status and living area indicated that adolescents who perform commuting to school passively are more likely (OR:1.50; IC95\%:1.12-2.01) being overweight. On the stratified analysis, the associations remain significant ( $p>0.05)$ only on girls. The reached results suggest that passive commuting to school is associated to overweight and obesity in adolescents.
\end{abstract}

\section{Keywords}

Body Mass Index; Sedentary; Adolescent.

\section{Introdução}

As prevalências de sobrepeso e a obesidade em crianças e adolescentes brasileiras têm se mostrado elevadas ${ }^{1-3}$. A preocupação com estas altas prevalências se

1 Universidade Federal do Pampa - Grupo de Pesquisa em Atividade Física e Saúde na Infância e Adolescência, Curso de Licenciatura em Educação Física, Uruguaiana, Rio Grande do Sul, Brasil. dá pelo fato de que o excesso de peso corporal está relacionado com o surgimento de fatores de risco para doenças crônicas não transmissíveis $(\mathrm{DCNT})^{4}$ e problemas psicossociais ${ }^{5}$ já nos anos da infância e adolescência. Além disso, estudos longitudinais sugerem que quando este quadro é instalado na infância tende a perdurar para a vida adulta ${ }^{6,7}$.

O ganho de peso corporal é um processo comple- 
xo envolvendo um conjunto de características relacionadas à genética, metabolismo, estilo de vida e ambiente em que os indivíduos estão inseridos ${ }^{8}$. Dentre aquelas relacionadas ao estilo de vida, o nível de atividade física pode desempenhar um importante papel no controle do peso corporal de adolescentes. As elevadas prevalências de sobrepeso e obesidade encontradas nesta população podem estar associadas ao declínio dos níveis da atividade física nos seus diferentes domínios (trabalho/escola, atividades domésticas, deslocamentos, e tempo livre) . $^{9}$

Com relação ao domínio deslocamento, tem sido investigado o transporte ativo como meio para ir à escola e suas possíveis associações com determinados indicadores de saúde, como peso corporal adequado e níveis elevados de atividade física ${ }^{10}$. No entanto, as informações disponíveis na literatura indicam um quadro de inconsistência nos resultados ${ }^{3,11,12}$ referentes às associações entre deslocamento ativo até a escola e o excesso de peso corporal. Novos estudos explorando estas associações devem ser conduzidos considerando análises estratificadas por sexo, pois as associações podem ocorrer de forma distinta entre rapazes e moças ${ }^{3,12}$.

Nesse contexto, considerando que o deslocamento ativo para a escola se constitui como uma estratégia para o aumento dos níveis de atividade física em escolares e, consequentemente, como um mecanismo auxiliar no controle do peso corporal, o presente estudo teve por objetivo identificar as associações entre a forma de deslocamento até a escola e frequência de sobrepeso e obesidade em adolescentes.

\section{Métodos}

\section{População e amostra}

Este estudo descritivo transversal de base escolar faz parte de uma série de investigações que estão sendo elaboradas com informações de um projeto maior intitulado "Atividade física e fatores associados em escolares de 10 a 17 anos de Uruguaiana, RS”. Este projeto foi submetido para análise e aprovado pelo comitê de ética em pesquisa da Universidade Federal do Pampa (protocolo 042/2010).

$\mathrm{O}$ banco de dados deste projeto conta com informações de 1.455 adolescentes de 10 a 17 anos de idade (50,9\% moças). O cálculo do tamanho da amostra foi realizado considerando: o número de alunos matriculados regularmente no ano de 2011 na faixa etária do estudo ( $\mathrm{n}=15.210$ ); prevalência de 50\% (considerando um estudo com múltiplos desfechos); intervalo de confiança de $95 \%$; erro aceitável de 3 pontos percentuais; e, percentual de perdas e recusas de $20 \%$. Com estes critérios o tamanho da amostra foi estimado 1.196 adolescentes.

O processo de seleção dos adolescentes foi probabilístico em múltiplas fases. Com o intuito de garantir a representatividade e proporcionalidade de alunos por regiões, inicialmente o município foi dividido em cinco áreas geográficas, quatro na zona urbana (quadrantes) e uma representativa da zona rural. Nove escolas da zona urbana e uma da zona rural foram sorteadas. Todas as escolas das cinco áreas tiveram a mesma chance de ser sorteadas.

Todos os alunos das turmas de quinta série (atual sexto ano) do ensino fundamental ao terceiro ano do ensino médio das dez escolas foram convidados a participar do estudo e receberam informações sobre sua realização. Todos os adolescentes que compareceram no dia agendado para a coleta de dados com o termo de consentimento livre e esclarecido assinado por um responsável e que manifestaram interesse em participar foram incluídos no estudo. Dentre estes, foram excluídos das análises os que estavam fora da faixa etária de interesse e aqueles que apresentassem algum tipo de deficiência física ou mental que os impedissem de praticar atividades físicas e/ou de compreenderem a realização das medidas. 


\section{Variáveis e procedimentos de coleta de dados}

Embora o banco de dados do projeto conte com outras variáveis, para a realização deste estudo foram utilizados os dados referentes ao índice de massa corporal (variável dependente) e à forma de deslocamento até a escola e o sexo (masculino ou feminino). Além destas, as variáveis sociodemográficas, idade (em anos completos), zona de moradia (urbana ou rural) e o nível socioeconômico (Classificação Econômica Brasil ${ }^{13}$ em cinco níveis de "A" a "E”) foram utilizadas para caracterizar a amostra.

Os valores de índice de massa corporal (IMC) foram calculados a partir da divisão dos valores de massa corporal em quilogramas $(\mathrm{kg})$ pela estatura em metros $(\mathrm{m})$ ao quadrado $\left[\mathrm{IMC}=\right.$ massa corporal $(\mathrm{kg}) /$ estatura $\left.(\mathrm{m})^{2}\right]$. A estatura e a massa corporal foram medidas conforme protocolos padronizados ${ }^{14}$. Os resultados do IMC foram categorizados com a utilização de pontos de corte específicos por sexo e idade ${ }^{15}$ em peso normal ou excesso de peso (somatório das classificações de sobrepeso e obesidade).

A forma de deslocamento até a escola fazia parte de uma série de informações contidas no instrumento (questionário) que foi entregue para que os adolescentes preenchessem. Este questionário foi elaborado pelos pesquisadores para atender aos objetivos do projeto. Para o deslocamento até a escola foi utilizada a questão: "Como você normalmente se desloca de casa para a escola e da escola para casa?", as opções de resposta eram: de carro/moto; de ônibus; transporte escolar; a pé; de bicicleta; outros (especifique). Foram considerados adolescentes com deslocamento ativo aqueles que relataram realizar o percurso a pé, de bicicleta, ou que assinalaram "outros" e descreveram alguma forma de deslocamento que dependesse de esforço físico. Com deslocamento passivo foram considerados aqueles que relataram utilizar algum tipo de veículo automotor. Todas as medidas foram realizadas por uma equipe de avaliadores (professores e bolsistas/estudantes de educação física) que passaram por uma capacitação. A coleta de dados ocorreu entre os meses de abril a novembro de 2011.

\section{Análise dos dados}

Inicialmente foi realizada a descrição da distribuição de frequência absoluta e relativa (proporções) das variáveis estudadas e seus respectivos intervalos de confiança de 95\% (IC95\%). Em seguida, analisou-se a associação bruta entre o deslocamento para a escola e as categorias do IMC, tendo as diferenças estatísticas sido avaliadas pelo teste do Qui-quadrado. A análise ajustada foi realizada utilizando-se a regressão logística binária. Para esta análise o sexo (análise envolvendo toda a amostra), a idade, o nível sócio econômico e a zona de moradia foram inseridos como variáveis de ajuste. As OR brutas e ajustadas e seus respectivos IC95\% foram calculados para estimar o tamanho do efeito das associações. Todas as análises de associação foram realizadas envolvendo toda a amostra e estratificadas por sexo. O nível de significância adotado foi de $5 \%$ e todas as análises foram realizadas no SPSS for Windows versão 20.0 .

\section{Resultados}

Foram selecionados 1.554 adolescentes. Destes, 41 não apresentaram o termo de consentimento livre e esclarecido ou não estiveram presentes no momento da coleta dos dados e 50 se recusaram a participar do estudo. Dos 1.463 adolescentes avaliados, oito foram excluídos das análises por estarem fora da faixa etária de interesse. Por fim, um total de 1.455 escolares (50,9\% do sexo feminino) participou 
do estudo. O nível socioeconômico C prevaleceu (63,9\%; IC95\%: 61,3-66,5) em relação aos níveis A/B (20,6\%; IC95\%: 18,4-22,8) e D/E (15,5\%; IC95\%: 13,5-17,5) e 9,2\% (IC95\%: 8,7-9,7) dos adolescentes eram residentes da zona rural.

Na tabela 1 são apresentadas as descrições das frequências de excesso de peso relativas à forma de deslocamento até à escola em toda a amostra e estratificada por sexo. A frequência de adolescentes com excesso de peso e que se desloca ativamente até a escola não diferiu entre os sexos ( $p>0,05)$.

TABELA 1 - Distribuição da frequência de adolescentes de 10 a 17 anos de acordo com o tipo de deslocamento até a escola e da classificação do índice de massa corporal. Uruguaiana/RS, 2011.

\begin{tabular}{|c|c|c|c|c|c|c|c|}
\hline \multirow{2}{*}{ Variável } & \multicolumn{2}{|c|}{ Toda amostra } & \multicolumn{2}{|r|}{ Masculino } & \multicolumn{2}{|r|}{ Feminino } & \multirow[t]{2}{*}{ Valor $p$} \\
\hline & $n$ & $\%(I C 95 \%)$ & $n$ & $\%(I C 95 \%)$ & $n$ & $\%(I C 95 \%)$ & \\
\hline \multicolumn{8}{|c|}{ Deslocamento até a escola } \\
\hline Ativo & 1082 & $75,7(73,5-77,9)$ & 540 & $76,6(73,5-79,7)$ & 542 & $74,8(71,6-78,0)$ & \multirow{2}{*}{$0,418^{*}$} \\
\hline Passivo & 348 & $24,3(22,1-26,5)$ & 165 & $23,4(20,3-26,5)$ & 183 & $25,2(22,0-28,4)$ & \\
\hline \multicolumn{8}{|c|}{ Índice de Massa Corporal } \\
\hline Normal & 990 & $72,2(69,8-74,6)$ & 504 & $73,7(70,4-77,2)$ & 486 & $70,6(67,2-74,0)$ & \multirow{2}{*}{$0,208^{*}$} \\
\hline Excesso de peso & 382 & $27,8(25,4-30,2)$ & 180 & $26,3(23,0-29,6)$ & 202 & $29,4(26,0-32,8)$ & \\
\hline
\end{tabular}

n - tamanho da amostra; \% - proporção da amostra; IC95\% - intervalo de confiança de 95\%; * teste Qui-quadrado.

A frequência de adolescentes com excesso de peso é significativamente maior entre os adolescentes que relataram realizar o deslocamento até a escola de forma passiva em toda a amostra $(\mathrm{p}<0,05)$ e entre as meninas ( $\mathrm{p}<0,05)$ (Figura 1). Quando a análise é realizada de forma ajustada, adolescentes que se deslocam de foram passiva até a escola tem mais chance (OR:1,50; IC95\%:1,12-2,01) de apresentarem excesso de peso. $\mathrm{Na}$ análise estratificada por sexo, as associações se mantêm significativas $(\mathrm{p}<0,05)$ apenas para o sexo feminino (tabela 2$)$.

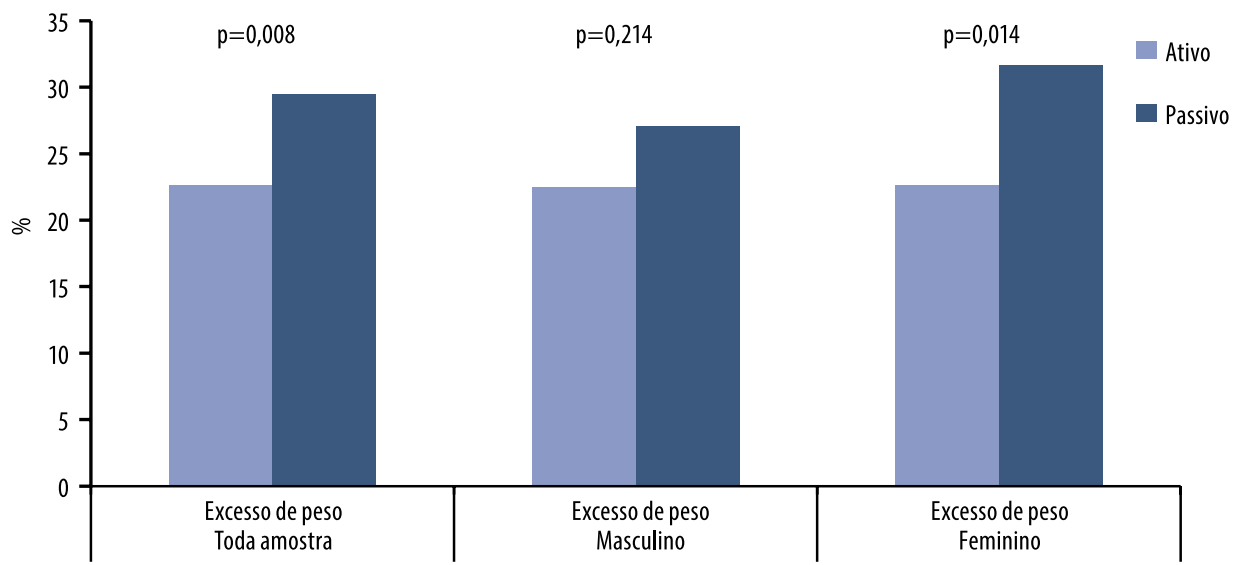

FIGURA 1 - Frequência de adolescentes de 10 a 17 anos com excesso de peso conforme a forma de deslocamento para a escola. Uruguaiana/RS, 2011.

Diferenças identificadas pelo teste Qui-quadrado. 
TABELA 2 - Odds Ratio bruta e ajustada entre índice de massa corporal e o deslocamento para a escola em adolescentes de 10 a 17 anos. Uruguaiana/RS, 2011.

\begin{tabular}{|c|c|c|c|c|c|c|}
\hline & \multicolumn{3}{|c|}{ Excesso de peso } & \multicolumn{3}{|c|}{ Excesso de peso } \\
\hline & $\begin{array}{c}\text { OR } \\
\text { bruta }\end{array}$ & IC95\% & $p$ & $\begin{array}{c}\text { OR } \\
\text { ajustada }\end{array}$ & IC95\% & $p$ \\
\hline \multicolumn{7}{|c|}{ Toda amostra } \\
\hline \multicolumn{7}{|c|}{ Deslocamento para a escola* $(n=1347)$} \\
\hline Ativo & 1 & - & - & 1 & - & - \\
\hline Passivo & 1,44 & $1,10-1,88$ & 0,008 & 1,50 & $1,12-2,01$ & 0,006 \\
\hline \multicolumn{7}{|c|}{ Masculino } \\
\hline \multicolumn{7}{|c|}{ Deslocamento para a escola ${ }^{* *}(n=675)$} \\
\hline Ativo & 1 & - & - & 1 & - & - \\
\hline Passivo & 1,28 & $0,87-1,89$ & 0,214 & 1,24 & $0,80-1,93$ & 0,332 \\
\hline \multicolumn{7}{|c|}{ Feminino } \\
\hline \multicolumn{7}{|c|}{ Deslocamento para a escola** $(n=672)$} \\
\hline Ativo & 1 & - & - & 1 & - & - \\
\hline Passivo & 1,59 & $1,10-2,30$ & 0,014 & 1,76 & $1,91-2,62$ & 0,005 \\
\hline
\end{tabular}

n: tamanho absoluto da amostra incluída nas análises ajustadas; OR: odds ratio; p: significância; *Ajustada para sexo, idade, nível socioeconômico e zona de moradia; ** Ajustada para idade, nível socioeconômico e zona de moradia.

\section{Discussão}

O principal resultado deste estudo foi identificar que adolescentes que se deslocam ativamente para a escola apresentam frequências de excesso de peso significativamente menor que aqueles que se deslocam passivamente. Os achados do presente estudo ficam fortalecidos diante do tamanho e do processo de seleção da amostra utilizada, que lhes conferem precisão e representatividade. Estas características aumentam o poder das análises e sua validade interna. Outra característica que pode ser destacada é o fato do estudo ter sido conduzido em uma cidade afastada dos grandes centros, na qual a maior parte das investigações nesta temática vem sendo conduzida. Ainda, a realização das análises estratificadas por sexo disponibiliza informações para a melhor compreensão das associações entre o a forma de deslocamento para a escola e a presença de excesso de peso em moças e rapazes.

Não obstante as forças do estudo, os resultados encontrados devem ser discutidos considerando algumas limitações. Por ser um estudo transversal não há possibilidade de atribuir relação de causa e efeito às associações encontradas. Considerando as variáveis analisadas, não é possível atribuir ao deslocamento até a escola de forma passiva a causa ou um fator determinante para os casos de excesso de peso. É necessário considerar que esta condição pode interferir na escolha dos adolescentes pela forma de deslocamento até a escola.

A forma de mensuração do transporte até a escola não considerou a distância percorrida ou o tempo para percorrê-la. Esta informação poderia indicar se há uma redução na frequência de excesso de peso conforme a distância percorrida até a escola aumenta. Por fim, o IMC foi classificado de acordo com a proposta de Cole et al. ${ }^{15}$. Esta proposta tem sido amplamente utilizada tanto no âmbito nacional ${ }^{1-3,16-18}$, quanto internacional ${ }^{19-22}$, facilitando a comparação dos resultados encontrados aos de outros estudos. Contudo, é preciso considerar que se outras propostas de classificação do IMC fossem utilizadas, prevalências de excesso de peso mais altas poderiam ser encontradas, pois alguns estudos ${ }^{18,19}$ tem indicado que as prevalências de sobrepeso e obesidade são menores quando a proposta de Cole et al. ${ }^{15}$ é utilizada. 
Cerca de três quartos da amostra relataram realizar o deslocamento para a escola ativamente. Esta frequência é superior às apresentadas por outros estu$\operatorname{dos}^{3,11,12,23,24}$, que variaram de $42 \%^{23}$ a $70 \%^{11}$. Este resultado pode ser explicado, ao menos em parte, devido à um dos critérios utilizados na rede pública (estadual e municipal) de ensino do município para a definição das escolas nas quais crianças e adolescentes são matriculadas. As crianças e adolescentes devem ser matriculadas preferencialmente nas escolas mais próximas a suas residências. Outra característica que pode auxiliar na compreensão da alta frequência de deslocamento ativo para a escola encontrada é o relevo plano do município em que o estudo foi realizado. Além disso, a escolha por deslocamentos passivos parece estar relacionada à preocupação dos pais com a distância a ser percorrida e a segurança ${ }^{25}$, características menos presentes em municípios de menor porte, como o do presente estudo.

Com relação às frequências de excesso de peso, os resultados são coerentes com aqueles encontrados por estudos realizados em cidades de diferentes regiões do país ${ }^{1-3,16-18}$ que utilizaram a mesma proposta de classificação do IMC, indicando que as prevalências tendem a ser mais altas no sul do país que nas demais regiões ${ }^{1,17,18}$. Tais resultados revelam uma situação preocupante, pois prejuízos à saúde decorrentes do excesso de peso corporal podem ser observados já nos anos da adolescência ${ }^{5,26}$. Além disto, adolescentes com excesso de peso tendem a permanecer nesta situação na idade adulta ${ }^{27}$.

No presente estudo adolescentes que relataram realizar deslocamento passivo para a escola tiveram mais chance de apresentar excesso de peso mesmo após ajuste para variáveis de confusão. No entanto, as informações disponíveis na literatura não são homogêneas sobre estas associações. Os estudos de Santos et al., ${ }^{12}$ e Rosaneli et al., ${ }^{23}$ não encontraram associação entre a forma de deslocamento até a escola e excesso de peso corporal em adolescentes. Por outro lado, os resultados das investigações conduzidas por Lopes e Silva ${ }^{11}$ e Benedet et al. $^{3}$, indicaram associações significativas entre as variáveis, tendo os adolescentes que relataram realizar deslocamento ativo até a escola frequências inferiores de sobrepeso e obesidade.

O panorama de inconsistência na possível relação entre a forma de deslocamento para a escola e o excesso de peso corporal em adolescentes também é encontrado no cenário internacional. Uma revisão sistemática ${ }^{28}$ analisou 18 estudos sobre a temática. Na metade deles não foram encontradas associações significativas entre as variáveis. Oito estudos encontraram associação entre o deslocamento passivo para a escola e excesso de peso corporal em pelo menos algum subgrupo (idade ou sexo) e um estudo encontrou associação entre o deslocamento ativo para a escola e maiores valores de IMC.

Apesar da heterogeneidade encontrada entre os resultados dos estudos analisados, deve-se considerar que o deslocamento ativo para a escola, além de ser uma alternativa para aumentar os níveis de atividade física ${ }^{10}$, parece contribuir para o controle do excesso de peso corporal em adolescentes ${ }^{3,11}$, sendo suportada pelos resultados do presente estudo. Dessa forma, a elaboração de estratégias para o combate ao sobrepeso e obesidade em adolescentes deve considerar a forma de deslocamento ativo até a escola como uma alternativa válida. Embora seja um processo complexo, envolvendo uma série de fatores, a elaboração dessas estratégias visando o aumento dos deslocamentos ativos entre os adolescentes, não só para a escola, mas para outros compromissos, devem ser pensadas envolvendo as áreas da educação, saúde, segurança e infraestrutura do poder público.

Em conclusão, os resultados do presente estudo indicaram que a prevalência de sobrepeso e obesidade entre os adolescentes analisados é elevada, corroborando os 
achados de outros estudos brasileiros. A maioria dos adolescentes realiza o deslocamento até a escola de forma ativa. Neste grupo, a frequência de sobrepeso e obesidade é menor do que entre aqueles que o realizam passivamente, indicando que o excesso de peso corporal está associado ao deslocamento não ativo para a escola.

\section{Contribuiç̧ão dos autores}

ARS participou da análise e interpretação dos dados, da redação do artigo e aprovou a versão final ser publicada. SG participou da análise e interpretação dos dados, da revisão crítica relevante do conteúdo intelectual e aprovou a versão final. MLAB e GGB participaram de todas as etapas do estudo e aprovaram sua versão final.

\section{Referências}

1. Tassitano RM, Barros MVG, Tenório MCM, Bezerra J, Hallal PC. Prevalência e fatores associados ao sobrepeso e à obesidade em adolescentes, estudantes de escolas de Ensino Médio de Pernambuco, Brasil. Cad Saúde Pública. 2009; 25(12): 2639-52.

2. Rech RR, Halpern R, Costanzi CB, Bergmann MLA, Alli LR, Mattos MP, et al. Prevalência de obesidade em escolares de 7 a 12 anos de uma cidade Serrana do RS, Brasil. Rev Bras Cineantropom Desempenho Hum. 2010;12(2): 90-7.

3. Benedet J, Assis MAM, Calvo MCM, Andrade DF. Excesso de peso em adolescentes: explorando potenciais fatores de risco. Rev Paul Pediatr. 2013;31(2):81-172.

4. Barreto SMB, Pinheiro ARO, Sichieri R, Monteiro CA, Batista F, et al. Análise da estratégia global para a alimentação, atividade física e saúde da Organização Mundial da Saúde. Epidemiol Serv Saúde. 2005;14 (1):81-68.

5. Silva D, Antunes H. Psychosocial correlates of overweight and obesity in infancy. Journal of Human Growth and Development. 2012;22(2):179-86.

6. Herman KM, Craig CL, Gauvin L, Katzmarzyk PT. Tracking of obesity and physical activity from childhood to adulthood: the Physical Activity Longitudinal Study. Int J Pediatr Obes. 2009;4(4):281-8.

7. Nakano T, Sei M, Ewis AA, Munakata H, Onishi C e Nakahori Y, Tracking overweigth and obesity im Japanese children; a six years longitudinal study. J Med Invest. 2010;57(12):114-23.

8. Oliveira LMP, Assis AMO, Silva MCM, Santana MLP, Santos NS, et al. Fatores associados a excesso de peso e concentração de gordura abdominal em adultos na cidade de Salvador, Bahia, Brasil. Cad de Saúde Pública. 2009;25(3):570-82.

9. Dollman J, Norton K, Norton L. Evidence for secular trends in children's physical activity Behaviour. Br J Sports Med. 2005;39:892-7.

10. Heelan KA, Donnelly JE, Jacobsen DJ, Mayo MS, Washburn R e Greene L. Active commuting to and from school and BMI in elementary school children - preliminary data. Child: Care, Health \& Development. 2005;31(3):341-9.

11. Silva KS, Lopes AS. Excesso de Peso, Pressão Arterial e Atividade Física no Deslocamento à Escola. Arq Bras Cardiol. 2008;91(2):93-101.

12. Santos CM, Júnior RSW, Barros SSH, Farias Júnior JC, Barros MVG. Prevalência e fatores associados à inatividade física nos deslocamentos para escola em adolescentes. Cad Saúde Pública. 2010;26(7):1419-30.

13. Associação Nacional de Empresas de Pesquisa. Critério de classificação econômica Brasil. São Paulo: Associação Nacional de Empresas de Pesquisa (dados com base no levantamento sócio econômico 2009); 2011.

14. Projeto Esporte Brasil. [citado 2015 mai 10]. Disponível em: http://www2.ufrgs.br/ proesp/

15. Cole TJ, Bellizzi MC, Flegal KM, Dietz WH. Establishing a standard definition for child overweight and obesity worldwide: international survey. BMJ. 2000;320(7244):1240-3.

16. Hobold E, Arruda M. Prevalência de sobrepeso e obesidade em estudantes: relações com nível socioeconômico, sexo e idade. Rev Bras Cineantropom Desempenho Hum 2015;17(2):156-64.

17. Guedes DP, Mendes RR. Crescimento físico e estado nutricional de escolares do Vale do Jequitinhonha, Minas Gerais, Brasil. Rev Bras Cineantropom Desempenho Hum. 2012;14(4):363-76. 
18. Pelegrini A, Silva DA, Gaya AC, Petroski EL. Comparison of three criteria for overweight and obesity classification in Brazilian adolescents. Nutr J. 2013;12:5.

19. Roberts KC, Shields M, de Groh M, Aziz A, Gilbert JA. Overweight and obesity in children and adolescents: results from the 2009 to 2011 Canadian Health Measures Survey. Health Rep. 2012;23(3):37-41.

20. Smetanina N, Albaviciute E, Babinska V, Karinauskiene L, Albertsson-Wikland K, Petrauskiene A, et al. Prevalence of overweight/obesity in relation to dietary habits and lifestyle among 7-17 years old children and adolescents in Lithuania. BMC Public Health. 2015;15:1001.

21.Zhu L, Chen Y, Ding L, Guo D, Wang L, Ren X, et al. Prevalence of overweight and obesity among secondary school children aged 14 to 18 years (china). Nutr Hosp. 2015;31(5):2006-10.

22. Symington EA, Gericke GJ, Nel JH, Labadarios D. The relationship between stunting and overweight among children from South Africa: Secondary analysis of the National Food Consumption Survey - Fortification Baseline I. S Afr Med J. 2015;106(1):65-9.

23. Rosaneli CF, Auler, F, Manfrinato CB, Rosaneli CF, Sganzerla C, Bonatto MG, Cerqueira MLW. Avaliação da prevalência e de determinantes nutricionais e sociais do excesso de peso em uma população de escolares: análise transversal em 5.037 crianças. Rev Assoc Med Bras. 2012;58(4):472-6.

24. Silva KS, Nahas MV, Peres KG, Lopes AS. Fatores associados à atividade física, comportamento sedentário e participação na Educação Física em estudantes do Ensino Médio em Santa Catarina, Brasil. Cad Saúde Pública. 2009;25(10):2187-200.

25. Davison KK, Werder JL, Lawson CT. Children's Active Commuting to School: Current Knowledge and Future Directions. Prev Chronic Dis. 2008;5(3):100-5.

26. López MS, Jensen ANM, Mir CN, Pianesi MEI, Manulak MA. Abdominal adiposity, insulin resistance, hypertension and urinary sodium in obese adolescents. Acta bioquím clín Latinoam. 2014;48 (3).

27. Singh AS, Mulder C, Twisk JW, Mechelen WV, Chinapaw MJ, Tracking of childhood overweigth into adulthood: a systematic review of the literature. Obes Rev. 2008;9(5):474-88.

28. Lee MC, Orenstein MR, Richardson MJ. Systematic Review of Active Commuting to School and Children's Physical Activity and Weight. J Phys Act Health. 2008;5(6):930-49.

ENDEREÇO PARA

CORRESPONDÊNCIA

GABRIEL GUSTAVO BERGMANN

gabrielbergmann@unipampa.edu.br
BR 472 - Km 592 - Caixa Postal 118

- Uruguaiana - RS - CEP: 97500-970

Universidade Federal do Pampa -

Campus Uruguaiana.

Fone: (55) 91993926
RECEBIDO 10/05/2015

REVISADO $18 / 12 / 2015$

$23 / 02 / 2016$

APROVADO $17 / 03 / 2016$ 